\title{
The Therapeutic Effect of Nigella Sativa Extract on Female Wistar Rats Vaginal Candidiasis Model
}

\author{
Muhammad Rusda ${ }^{1}$, M. Fidel Ganis Siregar ${ }^{1}$, Aznan Lelo ${ }^{2}$, Syafruddin Ilyas ${ }^{3}$, Ratna Akbari \\ Ganie $^{4}$, H.Kms. Yusuf Effendi ${ }^{5}$, Poppy Anjelisa ${ }^{6}$, R Lia Iswara ${ }^{7}$ \\ Obstetric and Gynecology Department, Faculty of Medicine, Universitas Sumatera Utara ${ }^{1}$ \\ Pharmacology Department, Faculty of Medicine, Universitas Sumatera Utara ${ }^{2}$ \\ Biology Departement, Faculty of Mathematics and Natural Sciences, Universitas Sumatera Utara ${ }^{3}$ \\ Clinical Pahology Depatment, Faculty of Medicine, Universitas Sumatera Utara ${ }^{4}$ \\ Obstetric and Gynecology Department, Faculty of Medicine, Universitas Sriwijaya \\ ${ }^{6}$ Faculty of Pharmacy, Universitas Sumatera Utara ${ }^{6}$ \\ Microbiology Department, Faculty of Medicine, Universitas Sumatera Utara ${ }^{7}$
} mrusdaharahap@yahoo.com

\begin{abstract}
Candidiasis is the most common fungal infection of humans caused by Candida albicans. Immediate and proper management of vaginitis candida must be done to prevent complications. Nigella sativa has pharmacological effects as antimicrobial, antiinflammatory, immune stimulation and anti-cancer properties. Nigella sativa was proven have antifungal effect in vitro, and this study was to assess the antifungal effects and possibility of in vivo therapy. This study is an analytical study that assesses the therapeutic effect of Nigella sativa in a rat vaginal candidiasis model. Subjects were 28 rats that had been inoculated with Candida albicans and divided into 4 groups: Nigella sativa group, fluconazole group, combination of Nigella sativa with fluconazole group and control group. Candida albicans colony was measured to assess the therapeutic effect of the treatment. There were no difference number of Candida albicans colony between all group before inoculation $(p=0,274)$ and after inoculation $(p=0,323)$. There were significant decreased number of Candida albicans colony on the 24 hours after the treatment between the three types of treatment with the control group (Nigella sativa group $p=0,046$; Fluconazole group $p=0,002$; Nigella sativa + fluconazole $p=0,002$ ). There was therapeutic effect of Nigella sativa by reducing the number of Candida albicans colonies.
\end{abstract}

Keywords: Fungal Inoculation, Pseudoestrus, Candida Albicans, Nigella Sativa, Vaginal Candidiasis, Fluconazole

\section{Background}

Candida albicans is the most common fungal infection of humans causing Candidiasis [1]. Complications of VVC that often occur is pelvic inflammatory disease which can ultimately cause infertility in sexually active women and chorioamnionitis which leading miscarriage or premature birth in women who are pregnant. Immediate and proper management of VVC must be done to prevent complications [2,3].

Nigella sativa is a member of the Ranunculaceae family. It has been used as a natural food and medicine which grows in Asia and the Middle East [4]. It contains Thymoquinone, 
Thymohydroquinone, Dithymoquinone and Thymol. Thymoquinone inhibits the oxygen cycle as inflammatory balance process with lipo-oxygenizes. Nigella sativa has pharmacological effects as antimicrobial, anti-inflammatory, immune stimulation and anti-cancer properties $[5,6]$.

The inhibitory effect of fungal by Nigella sativa extract has been proved by a study both in vitro and in vivo against some pathogenic fungal such as Candida albicans, dermatophytes, non-dermatophytes and some aflatoxin-producing fungi. It have shown Nigella sativa had a high inhibitory effect on candidiasis in rats in vivo and can reduce the number of Candida albicans 5-fold in the kidneys, 8-fold in the liver and 11-fold in the spleen. The antifungal effect of Nigella sativa treatment for single day on rats that had been inoculated with the Candida albicans apparently inhibited the growth of this pathogen [7,8].

There is no in vivo studies have been found in rats with vaginal candidiasis. Further research is needed to evaluate the anti-fungal activity of Nigella sativa in Vaginal Candidiasis. Asdadi had conducted the research to assess the Minimum Inhibitory Concentration (MIC) and Minimum Fungicidal Concentration (MFC) of Nigella sativa in Candida albicans [9].

\section{Research Method}

This research was an experimental study to assess therapeutic effect of Niggela sativa extract on vaginitis candida. This research was conducted in July - October 2019 on Animal House - Faculty of Mathematics and Natural Sciences, Universitas Sumatera Utara. Ethical clearance is obtained from Animal House - Faculty of Mathematics and Natural Sciences, Sumatera Utara University.

This study use 28 female Wistar rats (Rattis norvegicus sp) aged $2-3$ months with weight range of 101-240 grams that had fulfilled the inclusion and exclusion criteria. Before we do inoculation, we give Estradiol Valerate $2 \mathrm{mg}$ subcutaneously intraperitoneal 3 days before inoculation and 4 days after inoculation to make the rat in pseudoestrus state that needed to maintenance the Candida albicans, and prevent self-healing.

The breeding of Candida albicans was held in the microbiology laboratory, Universitas Sumatera Utara General Hospital. By swapping the vagina with a cotton swab dipped in ATCC 145033 McFarland Candida albicans cells, the inoculation was done.

The Nigella sativa extract was given from pharmacological laboratory, Faculty of Pharmacy, Universitas Sumatera Utara, using Sodium Carboxymethyl cellulose (Cmc Na) solvent in order to obtain the extract of Nigella sativa $5 \mathrm{mg} / \mathrm{mL}$.

We divided samples into 4 groups: 7 rats in the control group, 7 rats were given Nigella sativa extract $5 \mathrm{mg} / \mathrm{mL}$ with dose of $6.6 \mathrm{ml} / \mathrm{kg}$ of body weight, 7 rats were given $10 \mathrm{mg} / \mathrm{kg}$ of body weight, and 7 rats were given a combination of Nigella sativa extract $6.6 \mathrm{ml} / \mathrm{kg}$ of body weight and fluconazole $10 \mathrm{mg} / \mathrm{kg}$ of body weight.

After 24 hours inoculation, the therapy was given for 24 hours. The colonies of Candida albicans on the rats vagina was measure before inoculation and 24 hours after therapy. Vaginal smear samples were obtained, incubated for 48 hours on temperature $37^{\circ} \mathrm{C}$ and counted in the microbiology laboratory of the Universitas Sumatera Utara Hospital.

Data analyzed was using SPSS 22. We used Kruskal Wallis to assess differences in the four groups, $\mathrm{T}$ independent test and Mann Whitney to assess differences in the number of Candida albicans colonies in all four groups. 


\section{Results and Discussion}

Table 1. Colonies count of Candida albicans before inoculation

\begin{tabular}{ccccccc}
\hline & & \multicolumn{2}{c}{ Colonies count of Candida albicans, CFU/Plate } & \multirow{2}{*}{ p } \\
\cline { 3 - 6 } Group & $\mathbf{n}$ & Mean & SD & Median & Min - Max & \\
\hline A & 7 & 2,14 & 2,48 & 2 & $0-6$ & $0,274^{\mathrm{a}}$ \\
$\mathrm{B}$ & 7 & 10 & 18,68 & 0 & $0-51$ & \\
$\mathrm{C}$ & 7 & 0,57 & 0,79 & 0 & $0-2$ & \\
$\mathrm{D}$ & 7 & 0,14 & 0,38 & 0 & $1-1$ & \\
\hline
\end{tabular}

${ }^{a}$ Kruskal Wallis

Note: $\mathrm{A}=$ Nigella sativa group, $\mathrm{B}=$ fluconazole group, $\mathrm{C}=$ Nigella sativa group + fluconazole, $\mathrm{D}=$ control group.

The highest mean of number of Candida albicans colonies was found on the Fluconazole group with $10 \pm 18,68 \mathrm{CFU} /$ plate and lowest mean on the control group with $0,14 \pm 0,38$ $\mathrm{CFU} /$ plate. There was no difference of mean number colonies between four groups before inoculation with $\mathrm{p}=0,274$.

$=$ fluconazole group, $\mathrm{C}=$ Nigella sativa group + fluconazole, $\mathrm{D}=$ control group .

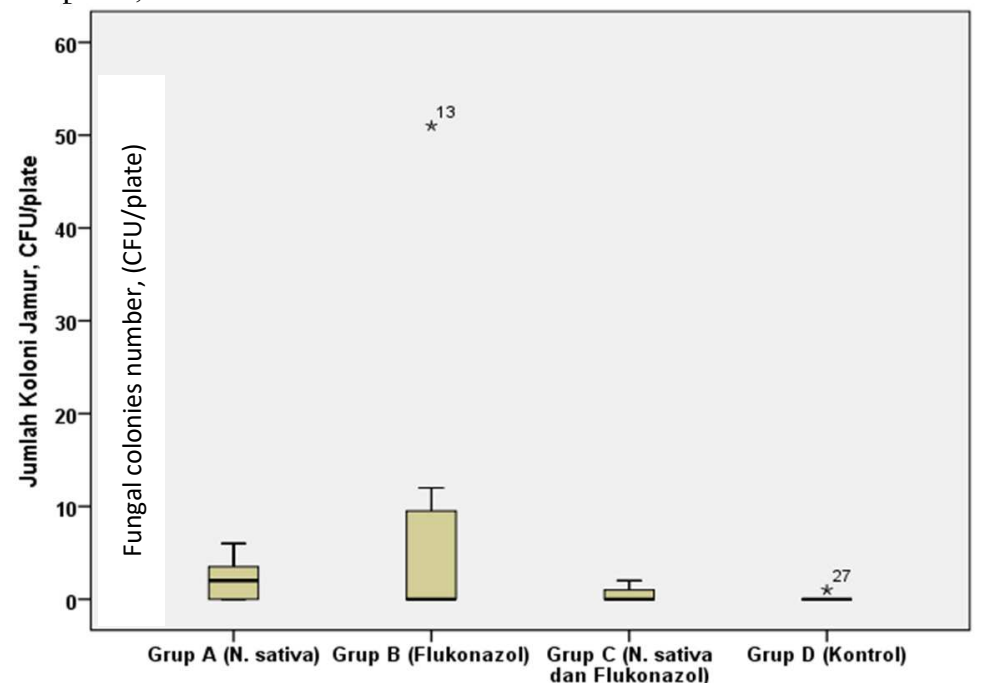

Fig 1. The number of Candida ai Group A Group B Group C

Table 2. Colonies Count of Candida albicans after inoculation

\begin{tabular}{|c|c|c|c|c|c|c|}
\hline \multirow{2}{*}{ Group } & \multirow{2}{*}{$\mathbf{n}$} & \multicolumn{4}{|c|}{ Colonies Count, CFU/Plate } & \multirow[b]{2}{*}{$\mathbf{p}$} \\
\hline & & Mean & SD & Median & Min - Max & \\
\hline A & 7 & 164,57 & 124,69 & 66 & $60-300$ & $0,323^{\mathrm{a}}$ \\
\hline B & 7 & 136,86 & 115,77 & 78 & $28-300$ & \\
\hline C & 7 & 210 & 95,67 & 243 & $56-300$ & \\
\hline D & 7 & 237,86 & 106,46 & 300 & $68-300$ & \\
\hline
\end{tabular}

${ }^{\mathrm{a}}$ Kruskal Wallis

Note: $\mathrm{A}=$ Nigella sativa group, $\mathrm{B}=$ fluconazole group, $\mathrm{C}=$ Nigella sativa group + fluconazole, $\mathrm{D}=$ control group. 
After the inoculation, remeasurement of vaginal colonies was done. The highest number of Candida albicans colonies was found on the control group with 237,86+106,46 CFU/plate and the lowest fungal colonies was in Nigella sativa group with $164,57 \pm 124,69$. There was no difference of mean number colonies between four groups after inoculation with $p=0,323$.

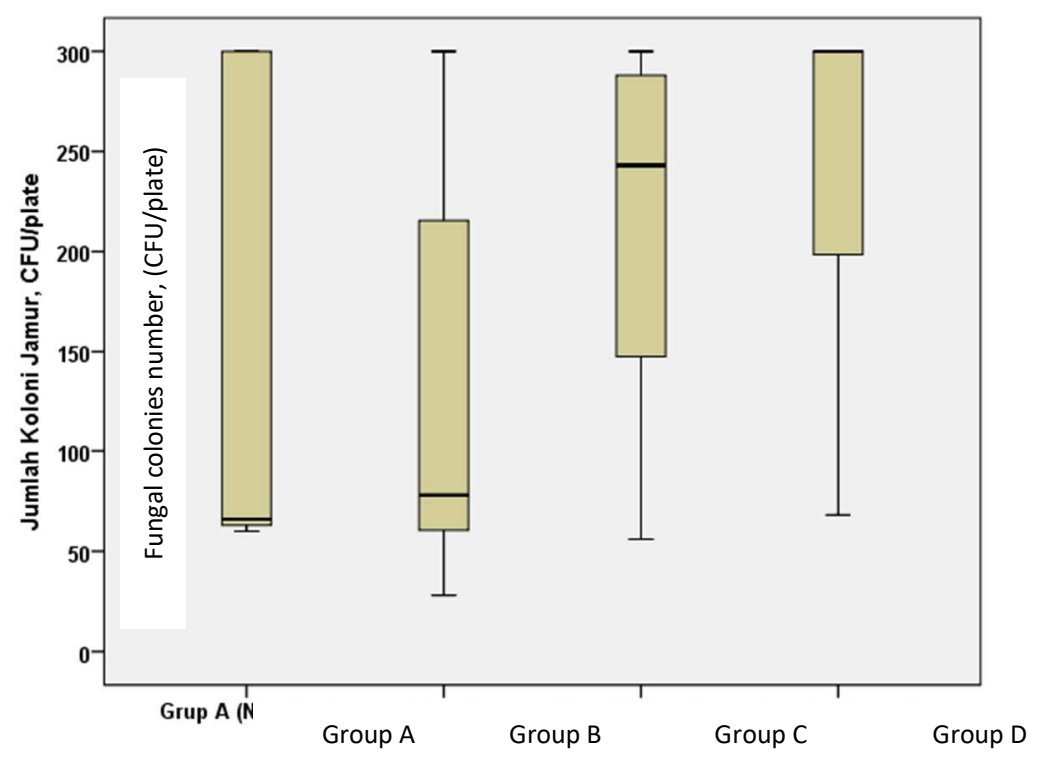

Fig 2. Colonies count of Candida albicans after inoculation. Note: $\mathrm{A}=$ Nigella sativa group, $\mathrm{B}=$ fluconazole group, $\mathrm{C}=$ Nigella sativa group + fluconazole, $\mathrm{D}=$ control group.

Table 3. Colonies Number of Candida albicans after 24 hours treatment

\begin{tabular}{|c|c|c|c|c|c|c|c|c|c|}
\hline \multirow{2}{*}{ Group } & \multirow{2}{*}{$\mathbf{n}$} & \multicolumn{4}{|c|}{ Colonies Number, CFU/Plate } & \multirow[b]{2}{*}{ p } & \multicolumn{3}{|c|}{ Post hoc } \\
\hline & & Mean & SD & Median & Min-Max & & B & $\mathrm{C}$ & D \\
\hline $\mathrm{A}$ & 7 & 88,29 & 99,63 & 53 & $19-300$ & $<0,001^{\mathrm{a}}$ & $0,002^{b}$ & $0,002^{b}$ & $0,046^{\mathrm{b}}$ \\
\hline B & 7 & 4,29 & 1,11 & 4 & $3-6$ & & & $0,107^{\mathrm{c}}$ & $0,001^{\mathrm{b}}$ \\
\hline $\mathrm{C}$ & 7 & 2,86 & 1,86 & 3 & $0-6$ & & & & $0,001^{\mathrm{b}}$ \\
\hline $\mathrm{D}$ & 7 & 229,29 & 120,89 & 300 & $43-300$ & & & & \\
\hline
\end{tabular}

${ }^{\mathrm{a}}$ Kruskal Wallis, ${ }^{\mathrm{b}}$ Mann Whitney, ${ }^{\mathrm{c}} \mathrm{T}$ Independent

Note: $\mathrm{A}=$ Nigella sativa group, $\mathrm{B}=$ fluconazole group, $\mathrm{C}=$ Nigella sativa group + fluconazole, $\mathrm{D}=$ control group

After 24 hours treatment, the therapeutic effect measured, we found that the lowest number of Candida albicans colonies belong to the combination group with $2,86 \pm 1,86$ CFU/plate, meanwhile the highest was in control group with $229,29 \pm 120,89 \mathrm{CFU} /$ plate. There was significant difference of the mean number of colonies between four groups after 24 hours of treatment $(p<0,001)$. There were significant difference between all four groups $(p<0,05)$ after Post Hoc Test. 


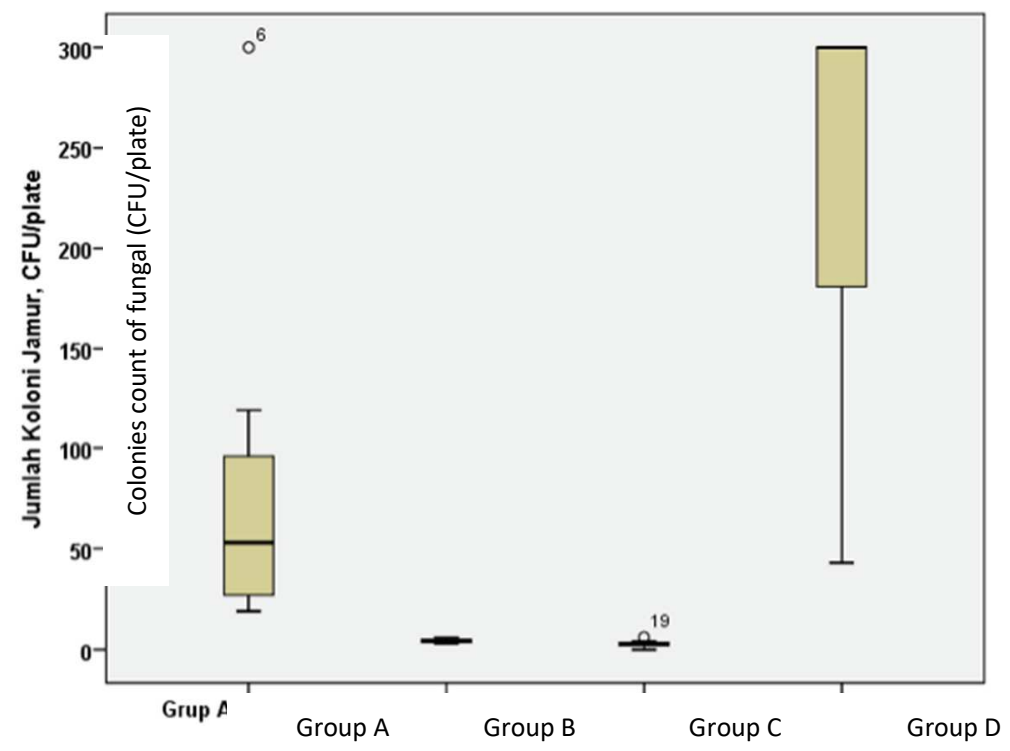

Fig 3. Colonies count of Candida albicans after 24 hours of treatment. Note : A = Nigella sativa group, $\mathrm{B}=$ fluconazole group, $\mathrm{C}=$ Nigella sativa group + fluconazole, $\mathrm{D}=$ control group.

\section{Discussion}

Creating, supporting and maintaining pathogens for vaginal candidiasis rat model were need a special treat. The Estradiol Valerate 2 - $5 \mathrm{mg}$ was administered subcutaneously intraperitoneal 3 days before inoculation and 4 days after to decrease rat immunity or creating a pseudoestrus situation. If needed, it can be repeated weekly [10].

The environment changing of vaginal candidiasis will quickly recover so that the infection will heal quickly without creating a pseudoestrus situation. ${ }^{12}$ The number of Candida albicans fungal colonies in all groups was increased (Nigella sativa: $2,14 \pm 2,48$ vs 164,57 $\pm 124,69$; Fluconazole: $10 \pm 18,68$ vs 136,86 $\pm 115,77$; Combination group: $0,57 \pm 0,79$ vs $210 \pm 95,67$ and Control group: $0,14 \pm 0,38$ vs $237,86 \pm 106,46)$. This indicated that the rats were successfully became vaginitis candida model.

Nigella sativa Linn, Ranunculaceae family, was grown in many parts of the world, especially the Mediterranean region, North Africa, the Middle East and parts of Asia and used by herbal therapy for many diseases [7].

The component of Nigella sativa were thymoquinquinone (TQ), alcaloid (nigellicines and nigelledine), saponins (alpha-hederin), flavonoids, proteins, fatty acids, and many others, which have various therapeutic effects [12].

Nigella sativa extract have the strongest antifungal effect against various pathogenic fungal strains, including methanol, ethanol and chloroform extracts [6]. Even water extracts from Nigella sativa seeds showed an inhibitory effect on candidiasis in rat [13]. From the results of this study the administration of Nigella sativa extract with Sodium Carboxymethyl cellulose can reduce the number of vaginal colonies of the Candida albicans $(\mathrm{p}=0,002)$. 
On previous study on systemic candidiasis, therapeutic effect of Nigella sativa in the 24hour observation after administration can reduce 5 -fold the number of candida organisms in the kidney, 8-fold in the liver and 11-fold in the spleen [14][15][16].

Based on the treatment groups comparison, all the three treatment groups include were Nigella sativa extract group, fluconazole group and combination of Nigella sativa and fluconazole extract group can reduce number of fungal colonies compared with the control group $(\mathrm{p}=0,046 ; \mathrm{p}=0,002 ; \mathrm{p}=0,002)$.

\section{Conclusions}

Based on this study, we conclude that the therapeutic effect of Nigella sativa reduce the number of Candida albicans colonies.

\section{Reference}

[1] Ackhar, M.J. Candida infections of the genitourinary tract. Clinical Microbiology Reviews. (2010).

[2] Al-ahmadey, Z. Z. and Mohamed, S. A. Vulvovaginal candidiasis: Agents and its virulence factors. Microbiology research international. (2010).

[3] Sahoo S et al. Prevalence of Vulvovaginal Candidiasis in Sexually Active Females with Antijamur Susceptibility Patterns of the Isolates. International Journal of Clinical and Biomedical Research. (2018).

[4] Ahmad, A. et al. A review on therapeutic potential of Nigella sativa: A miracle herb', Asian Pacific Journal of Tropical Biomedicine. (2013).

[5] AL-Beitawi, N. A., EL-Ghousein, S. S. and Nofal, A. H. Replacing bacitracin methylene disalicylate by crushed Nigella sativa seeds in broiler rations and its effects on growth, blood constituents and immunity. Livestock Science. Elsevier B.V. (2009).

[6] Aljabre, S. H. M. et al. Antidermatophyte activity of ether extract of Nigella sativa and its active principle, thymoquinone. Journal of Ethnopharmacology. (2005).

[7] Candel, F. J. et al. Update on management of invasive candidiasis. Revista Espanola de Quimioterapia. (2017).

[8] Chaieb, K. et al. Antibacterial activity of Thymoquinone, an active principle of Nigella sativa and its potency to prevent bacterial biofilm formation. BMC. (2011).

[9] De Bernardis, F., G. Santoni, M. Boccanera, E. Spreghini, D. Adriana, L. Morelli, and A. Cassone. Local anticandidal immune responses in a rat model of vaginal infection by and protection against Candida albicans. Infection and Immunity. (2000).

[10] Fidel, PL., Cutright, J. and Steele, C. Effects of Reproductive Hormones on Experimental Vaginal Candidiasis. Infection and Immunity. (2000).

[11] Martinez, A. et al. Antigungal efficacy of GM237354 and GW471558 in experimental model of oral and vuvlovaginal candidiasis in immunocompromised rats', Antimicrobial Agents and Chemotherapy. (2001). 
[12] Mohammed, A. E.-N. A. and Al-Suwaieg, S. B. Effects of Nigella sativa on mammals' health and production', Adv. Anim. Vet. (2016).

[13] Rafati, S., Niakan, M. and Naseri, M. Anti-microbial effect of Nigella sativa seed extract against staphylococcal skin Infection. Medical Journal of the Islamic Republic of Iran, (2014).

[14] Salem, M. L. Immunomodulatory and therapeutic properties of the Nigella sativa L. seed. International Immunopharmacology. (2005).

[15] Utami AT. Nigella sativa Linn. and Lower Urinary Tract Infection Treatment. Journal of Surgery Pathology Diagnosis. (2018).

[16] Sokri, H. A review on the inhibitory potential of Nigella sativa against pathogenic and toxigenic fungi. Avicenna journal of phytomedicine. (2016). 